\title{
Quantification of Cell Adhesion Force with Atomic Force Microscope
}

\author{
CHEN Sheng ${ }^{1, a, *}$, XIE Hui ${ }^{1, b}$ \\ ${ }^{1}$ State Key Laboratory of Robotics and System, Harbin Institute of Technology, Yikuang Street, \\ Harbin 150036, China \\ achensheng263@163.com, biehuimmp@163.com \\ *CHEN Sheng
}

Keywords: atomic force microscope; probe; single-cell force spectroscopy; adhesion force.

Abstract: Cell adhesion to cells or substrates is the basis for cell colonization and survival. Based on atomic force microscope (AFM), a single probe was used to quantitatively measure the adhesion of different cell types. The self-made pneumatic control system produces negative pressure to fix the cells, replacing the traditional chemical fixation method, reducing the fixation time from about $30 \mathrm{~min}$ to a few seconds, and a single probe can test about 15 cells, greatly improving single-cell force spectroscopy test speed. As a result, statistical data can be quickly recorded, the range of cells tested can be expanded, and the physiological activity of the cells during the test is preserved. In this paper, we studied the adhesion of three different mammalian cells to polystyrene substrates, and indicated that the adhesion forces depend on the probe retraction speed and the contact time: the adhesion forces are proportional to the logarithm of the probe retraction speed and increase with increasing contact time.

\section{Introduction}

The cells interact with the external environment by regulating the expression of molecular proteins on the cell membrane. The molecular proteins on the cell membrane are combined with the molecular proteins in the external environment in the form of receptor-ligand to carry out signal exchange and regulate the adhesion of cells to the external environment force. Cell adhesion to the surrounding environment is involved in signal transmission between cells and the surroundings, material transport, energy collection, embryo and tissue development, tumor metastasis, viral and bacterial infections, and therefore is very important in cell physiology. Optical microscopy and flow chamber methods only obtain qualitative and semi-quantitative adhesion data, while the first set of quantitative data is measured by optical tweezers, magnetic tweezers, or micropipettes [1-4]. In recent years, the information about cell adhesion has been updated based on the force spectrum of atomic force microscopy (AFM), especially at the single molecule level and the nanomaterial properties of the cells [5]. Single-cell force spectroscopy usually fixes the cells irreversibly by the chemical modification of the cantilever, resulting in the need for a new probe to be chemically modified and calibrated for each cell to be measured, greatly reduced effectiveness. The chemical fixation process of cells requires a significant amount of time (usually 30 minutes [6]) and may alter the physiological characteristics of the cells [7]. In addition, the cells to be tested must be chemically immobilized, and this usually requires a wealth of experience to find the optimal 
chemical modification of different cells. The binding force between the cell and the cantilever also limits the force range of the single-cell force spectroscopy, which leads to the fact that the method can only be applied before the contact force goes beyond the measurable range [8]. In this paper, we describe and apply a method that allows single cell force spectroscopy to use a probe to measure the adhesion of multiple cells, greatly reducing the time required to obtain statistically relevant data. In addition, the time required for cell fixation of the cantilever beam is shorter (several seconds) compared to conventional single cell force spectroscopy and allows cells to contact the substrate for extended periods of time. Based on AFM, a series of dynamic and long-term adhesion tests were performed on different mammalian cells using improved single-cell force spectroscopy, demonstrating the universality and wide applicability of the method.

\section{Method and Materials}

\subsection{Single-cell Force Spectroscopy}

Single-cell force spectroscopy is accompanied by the development of atomic force microscopy, which uses a cantilever probe as a force sensor to detect weak changes. As shown in Figure 1 (A), the atomic force microscope approaches the probe in contact mode and contacts it with a certain pressure. After maintaining the pressure for several tens of minutes, the chemically modified probe tip fixes the cells to the probe tip. Then, as shown in Figure 1 (B), when the cells are pulled by retracting the probe, the cells will also generate a pulling force on the probe tip depending on the force and the reaction force, causing the probe cantilever to deflect. The incident laser is irradiated at the end of the probe cantilever, and the outgoing beam of the laser beam is shifted due to the deflection of the probe. The offset is received by the Position Sensitive Device (PSD). The output voltage of the PSD circuit reflects the deflection of the cantilever beam. In the initial stage of pulling the cells, the pull of the probe on the cells is not sufficient to overcome the adhesion of cells to the substrate. As the distance between the probe and the substrate increases, the deformation of the cantilever beam increases, resulting in a continuous drop in the output voltage of the detection circuit. The tension applied to the cell becomes larger and the tension will gradually overcome the adhesion. At the moment when the tension is greater than or equal to the cell and substrate adhesion, the cells begin to be lifted, the deflection of the cantilever will gradually recover, the output voltage begins to rise and eventually stabilize at a certain value. The output voltage-distance curve of the probe can be transformed into a force-distance curve (Figure 3), and the maximum force and maximum separation distance can be obtained by analyzing the force-distance curve. The relationship between the output voltage and the force is:

$$
F=\frac{k}{S} \times V
$$

where $F$ is the adhesion of the cell to the substrate, $k$ is the normal stiffness of the probe cantilever, $V$ is the PSD output voltage, $S$ is the probe cantilever normal sensitivity, that is, PSD output voltage variation caused by the probe cantilever unit normal deformation. 

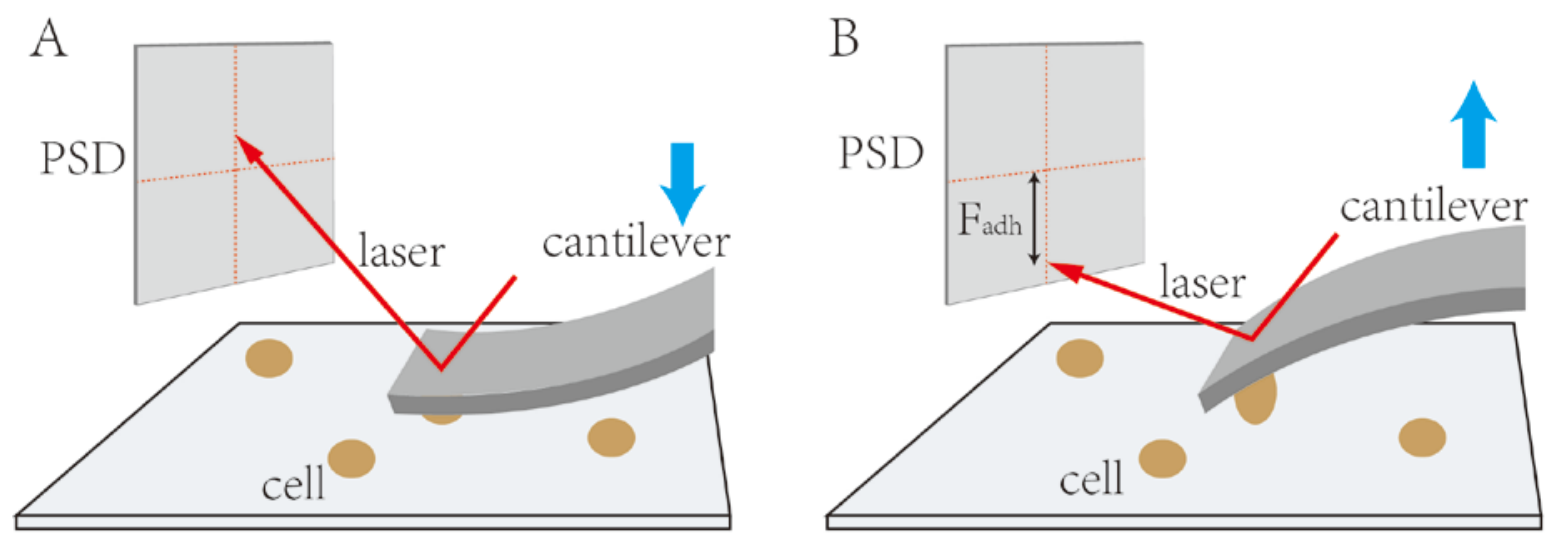

Figure 1 AFM-based single-cell force spectroscopy.

This study has improved the single-cell force spectroscopy based on AFM: instead of the original method of chemical modification, the underpressure generated by the pressure control system fixes the cells on the hollow probe tip, as shown in Figure 2. Compared to the original method, the method of cell fixation can be completed in a few seconds, greatly reducing the time it takes to fix the cells on the cantilever. The overpressure generated by the pneumatic device can release the fixed cells, so that a single probe can measure the adhesion of multiple cells, without the need for a number of new probes for chemical modification and calibration, improve the efficiency of the force measurement. In addition, the underpressure in the hollow probe exceeds the binding force of the chemical modification to the cells, so this method allows the cells to contact the substrate for a long time, and the measurement range of the adhesion force is wider.

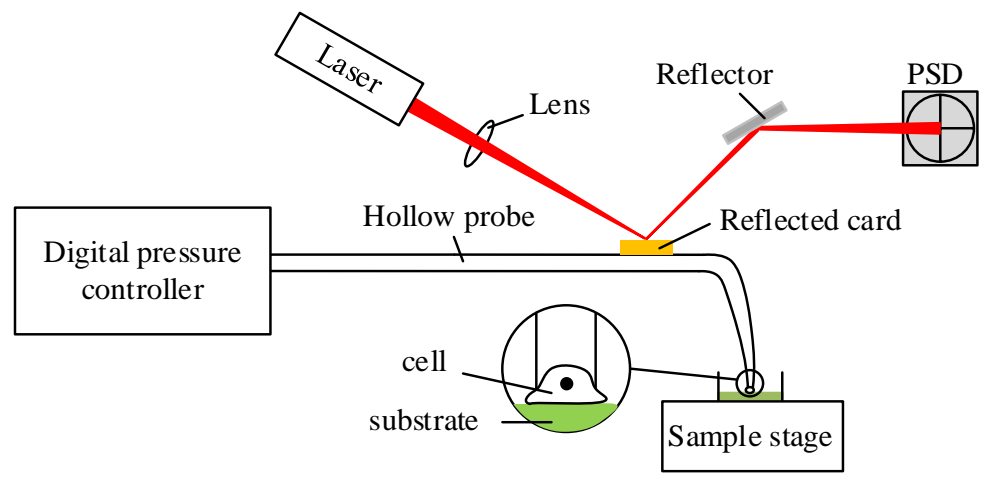

Figure 2 Improvement of AFM-based single-cell force spectroscopy.

\subsection{Cell and Substrate}

The cells measured in this paper were 3T3 cells, HeLa cells and HepG2 cells. Cells were maintained in growth medium consisting of eagle's media supplemented with $15 \%$ fetal bovine serum at $37^{\circ} \mathrm{C}$ in a humidified atmosphere with $5 \% \mathrm{CO}_{2}$. Before the adhesion measurement, the cells were rinsed three times with PBS buffer to adjust the cell concentration so that the appropriate amount of cells was obtained on the substrate. The substrate used for the experiment was polystyrene (PS).

\subsection{Pressure Controller}

To cell aspiration and release, the pressure controller must be able to produce a controlled 
positive and negative pressure. The controller contains a vacuum pump and compressed air machine to produce a relatively stable positive and negative pressure, and then absorb cells and release cells. When a probe is needed to draw cells, the negative pressure airway acts; when the probe is needed to release the cell, the positive pressure airway works. In addition, if the negative pressure within the probe is not stable, then the distance between the sucked cell and the probe will continue to change, so that the cantilever shakes up and down, resulting in measurement results are not accurate. In order to improve the accuracy of the experimental results, the controller must be able to provide a stable negative pressure. So a precision vacuum valve is essential to achieve a stable negative pressure output.

\subsection{Atomic Force Microscope}

The AFM system consists of an overhead optical microscope, a side view optical microscope, a temperature controller, a CO2 concentration controller and a light lever force feedback system and a drive positioning system. The overhead optical microscope is used to find the appropriate cells and observe the orientation between the sample and the probe. The side view optical microscope is used to observe the adhesion of the cells to the substrate and the fixation of the cell and probe. Light lever force feedback system consisting of lasers, lenses, probes, four quadrant photoelectric converters (PSD), can measure the size of the weak force. The coarse positioning and fine positioning of the probe are carried out respectively by the micrometer stage I (displacement: 13 $\mathrm{mm} \times 13 \mathrm{~mm} \times 13 \mathrm{~mm}$, accuracy: $10 \mathrm{~nm}$ ) and the nanometer stage I (displacement: $10 \mu \mathrm{m} \times 10 \mu \mathrm{m} \times$ $10 \mu \mathrm{m}$, accuracy: $0.1 \mathrm{~nm}$ ). The sample stage was supported by a nanometer stage II (displacement: $75 \mu \mathrm{m} \times 75 \mu \mathrm{m} \times 50 \mu \mathrm{m}$, accuracy: $0.1 \mathrm{~nm}$ ) and driven. The temperature controller and the CO2 concentration controller are used to control the temperature and $\mathrm{CO} 2$ concentration in the system to ensure that the cell activity is not affected.

\section{Results and Discussion}

\subsection{The Maximum Adhesion of the Three Cell Types}

In this paper, we used improved single cell force spectroscopy to test the adhesion of different cells to PS substrates. Prior to testing, all cells had grown on the PS substrate for 10 hours. After the measured cell were selected by optical microscopy, the probe was allowed to approach the cell on substrate at a speed of $1 \mu \mathrm{m} / \mathrm{s}$ in contact mode at a set point of $50 \mathrm{nN}$. When the probe is in contact with the cells, the pressure inside the probe is controlled to $-100 \mathrm{kPa}$ and the probe is held for 3 seconds to keep the cells fixed at the probe tip. The probe was retracted at a speed of $1 \mu \mathrm{m} / \mathrm{s}$ and the retraction distance was $40 \mu \mathrm{m}$. The force-displacement curve of the whole process was obtained. When the probe retraction is completed, the pressure inside the probe is controlled by the pneumatic device to release the measured cells at $100 \mathrm{kPa}$, so that the next test cell can be searched and sucked. Figure 3 shows the force-displacement curve of a HeLa cell on the substrate. The red curve is the approach curve of the probe tip. The blue curve is the curve of the cell adhesion to the substrate and the displacement of the probe tip. From the curve, it can be seen that the adhesion force is $411 \mathrm{nN}$, the separation distance is $28 \mu \mathrm{m}$, and many jump points (identified as Jumps) and the step surfaces (identified as Tethers ) appear after separation of $13 \mu \mathrm{m}$. In this study, 15 HeLa cells, 12 HepG2 cells and 14 3T3 cells were tested for their adhesion to the substrate using three probes, respectively. The results are shown in Table 1, which demonstrates that the improved single cell force Spectrum has a wide range of applicability. 


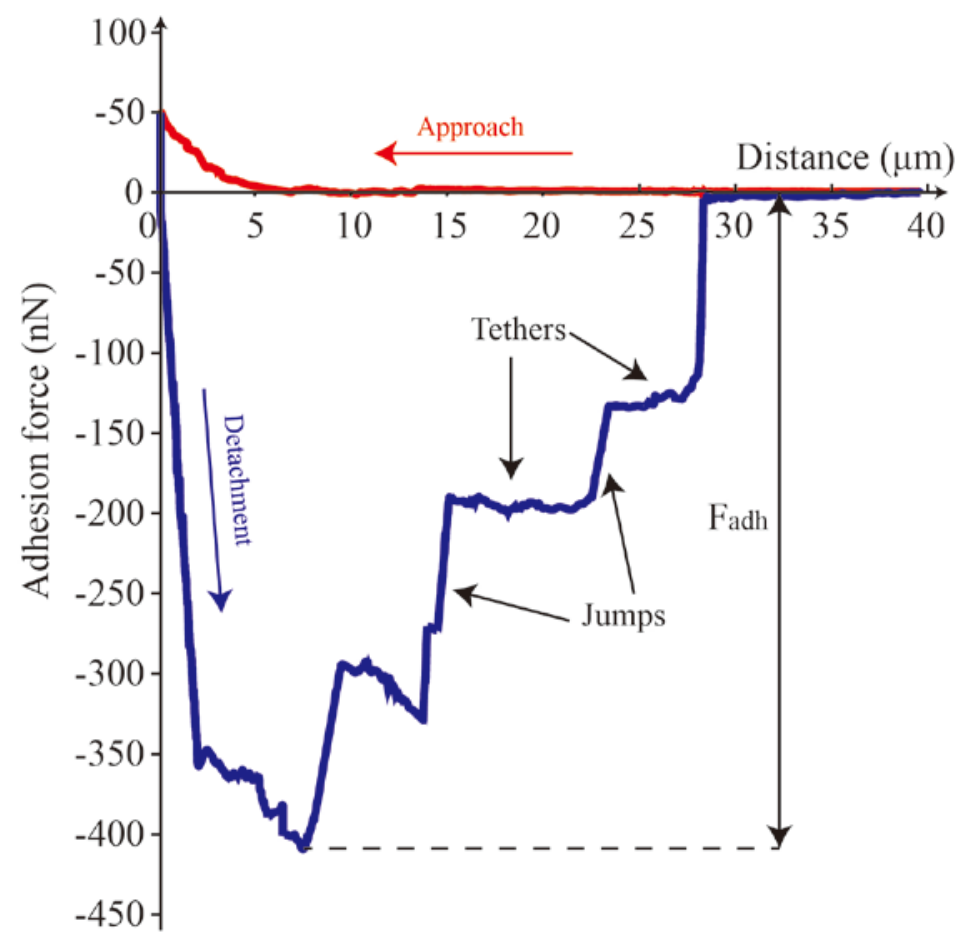

Figure 3 The force-distance curve of a HeLa cell on PS substrate.

Table 1 Adhesion forces of different cell types on PS substrate.

\begin{tabular}{|c|c|c|}
\hline Cell type & Mean/nN & \pm Standard error/ $\mathrm{nN}$ \\
\hline HeLa & 463 & 85 \\
\hline 3T3 & 412 & 36 \\
\hline HepG2 & 486 & 57 \\
\hline
\end{tabular}

\subsection{The Effect of Probe Retraction Speed on Adhesion}

Prior to testing, HeLa cells had grown on the PS substrate for 10 hours. After the measured cell were selected by optical microscopy, the probe was allowed to approach the cell on substrate at a speed of $1 \mu \mathrm{m} / \mathrm{s}$ in contact mode at a set point of $50 \mathrm{nN}$. When the probe is in contact with the cells, the pressure inside the probe is controlled to $-100 \mathrm{kPa}$ and the probe is held for 3 seconds to keep the cells fixed at the probe tip. The probe was retracted at a speed of $0.3 \mu \mathrm{m} / \mathrm{s}, 0.5 \mu \mathrm{m} / \mathrm{s}, 1 \mu \mathrm{m} / \mathrm{s}$, $2 \mu \mathrm{m} / \mathrm{s}$ and $4 \mu \mathrm{m} / \mathrm{s}$, respectively. The retraction distance was $40 \mu \mathrm{m}$, and the force-displacement curve of the whole process was obtained. In this study, 13 cells were used to test cell adhesion at a retraction speed of $0.3 \mu \mathrm{m} / \mathrm{s}, 11$ cells were used to test cell adhesion at a retraction speed of $0.5 \mu \mathrm{m}$ / s, and 14 cells were used to test cell adhesion at a retraction speed of $1 \mu \mathrm{m} / \mathrm{s}, 16$ cells were used to test cell adhesion at a retraction speed of $2 \mu \mathrm{m} / \mathrm{s}$, and 12 cells were used to to test cell adhesion at a retraction speed of $4 \mu \mathrm{m} / \mathrm{s}$. A new probe was used for each group of experiments. The results of the test are shown in Figure 4, which shows that the cell adhesion is proportional to the logarithm of the probe retraction speed and conforms to the Bell-Evans model expression. 


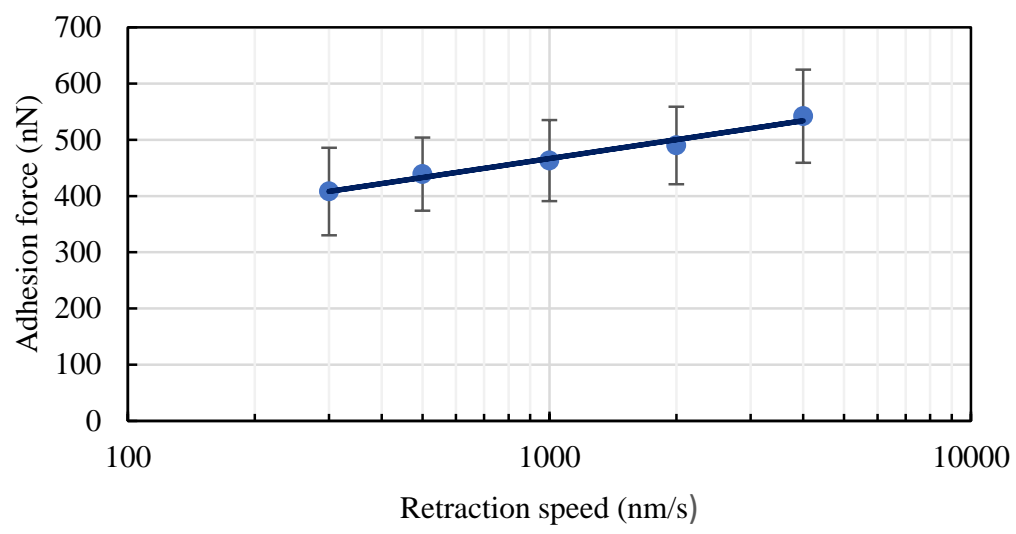

Figure 4 The relationship between adhesion force and retraction speed.

\subsection{The Effect of the Contact Time on Adhesion}

Prior to testing, HeLa cells were suspended in the culture medium and were not contacted with the substrate. After the cells were selected by optical microscopy, the probe was allowed to slowly approach the suspended cells. When the probe is in contact with the cells, the pressure inside the probe is controlled to $-100 \mathrm{kPa}$ and the probe is held for 3 seconds to keep the cells fixed at the probe tip. The probe was driven at a speed of $1 \mu \mathrm{m} / \mathrm{s}$ with the cells, slowly approaching and contacting the substrate in a contact mode at a set point of $50 \mathrm{nN}$. The contact times were $10 \mathrm{~s}, 30 \mathrm{~s}$, $50 \mathrm{~s}, 70 \mathrm{~s}, 90 \mathrm{~s}$, respectively. Then the probe was retracted at a speed of $1 \mu \mathrm{m} / \mathrm{s}$ until the cells were separated from the substrate, and the force-displacement curve of the whole process was obtained. In this study, 13 cells were used to test cell adhesion at a contact time of $10 \mathrm{~s}, 11$ cells were used to test cell adhesion at a contact time of $30 \mathrm{~s}, 14$ cells were used to test cell adhesion at a contact time of $50 \mathrm{~s}, 16$ cells were used to test cell adhesion at a contact time of $70 \mathrm{~s}, 12$ cells were used to test cell adhesion at a contact time of $90 \mathrm{~s}$. A new probe was used for each group of experiments. The results of the test are shown in Figure 5, which shows that the cell adhesion increases with the increase of the contact time. With the increase of contact time, more receptor molecules (such as integrins) on the cell surface combine with the ligand molecules of the external matrix to form more molecular bonds, resulting in increased adhesion between the cells and the substrate. As the total number of receptors on the cell membrane is conserved, the tendency of adhesion decreases with increasing contact time, and eventually reaches a steady state.

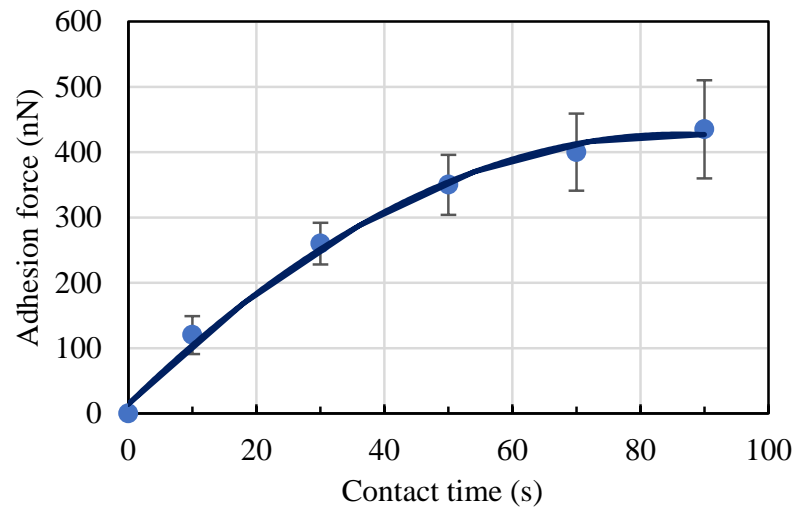

Figure 5 The relationship between adhesion force and contact time. 


\subsection{Discussion}

At present, the traditional single cell force spectroscopy is difficult to test the cell maximum adhesion with long contact time, even if Hosseini et al succeeded to prove the relationship between cell adhesion and time, but in the case of longer contact time, it is easy to test failed[9]. The reason is that the adhesion between cells and the substrate exceeds the chemical adhesion of cells to the cantilever. Therefore, in the traditional single cell force spectroscopy, the cell contact time with the substrate cannot be more than 1 hour. Although Weder et al. succeeded to make the cell interacts specifically with the cantilever through a fibronectin-modification to enhance the binding strength of the cantilever to the cell, extending the cell-to-substrate contact time to 24 hours, the adhesion of cells to protein-modified cantilever beams takes 30 minutes or more[10]. In order to interact specifically with fibronectin, the cells will alter their gene expression to diffuse the adhesion receptor in the membrane surface, and altering the structure of the cell surface. In this study, improved single-cell force spectroscopy not only tests cells in contact with the substrate for a long time, but also avoids the effects of chemical action on the cell membrane. In addition, a single probe in a conventional method can only test one cell, and each probe needs to be calibrated before testing and the time required to fix the cells is greater than 30 minutes. In the improved method, a single probe can test about 15 cells, and the time required to fix the cells is only a few seconds, increasing the test speed and reducing the cost of the probe.

In addition, it can be seen from the force-distance curve obtained that the adhesion curve is composed of many jumping points and step surfaces during the process of cell detachment from the substrate. The jump points are formed by the ruptures of molecular bonds between receptors on cell membrane and the ligands on substrate. The reason for the formation of the step surface may be that when the receptor-ligand molecule is not easily broken, the receptor in the cell membrane is stretched while the cell is away from the substrate, causing the cell membrane to be pulled out like a nanotube. We assume that there is no cytoskeleton in the nanotubes, the adhesion energy is zero. Then when the nanotube forms, the force-distance curve remains essentially unchanged, forming a step force.

\section{Conclusion}

Based on AFM, we used vacuum negative pressure instead of chemical modification to fix the cells on the probe, and improved the traditional single cell force spectrum method. The improvement accelerates the speed of immobilized cells, reduces the time required for multiple probe calibration, enhances the adhesion of cells to the probe, broads the measurement range of cell adhesion, and meanwhile, avoids the effect of chemical modification on the cell membrane, makes rapid and serial quantification of adhesion force possible. In this paper, an improved method was investigated to test different animal cells, proving a wide range of applicability. Through several sets of tests about different retraction speeds, we found that the cell adhesion is proportional to the logarithm of the probe retraction speed and conforms to the Bell-Evans model expression. Through several sets of tests about different contact times, we found that the cell adhesion grows with the contact time and the tendency of adhesion growth decreases with contact time, and eventually reaches a steady state.

The results obtained in this study will provide more reference for the study of cell adhesion properties, and the application of the equipment and methods in this study will provide new tools and technical options for the study of nano biomechanics. 


\section{References}

[1] An, Y. H., Friedman, R. J. (1997) Laboratory methods for studies of bacterial adhesion. Journal of Microbiological Methods, 30: 141-152.

[2] Ashkin, A., Dziedzic, J. M. (1987) Optical trapping and manipulation of viruses and bacteria. Science, 235: 1517-1520.

[3] Walter, N., Selhuber, C., Kessler, H., et al. (2006) Cellular unbinding forces of initial adhesion processes on nanopatterned surfaces probed with magnetic tweezers. Nano Letters, 6: 398-402.

[4] Shao, J. Y., Xu, G., Guo, P. (2004) Quantifying cell-adhesion strength with micropipette manipulation: principle and application. Front Biosci, 9: 2183-2191.

[5] Binnig, G., Quate, C. F., Gerber, C. (1986) Atomic Force Microscope. Phys Rev Lett, 56: 930-933.

[6] Weder, G., Blondiaux, N., Giazzon, M., et al. (2010) Use of force spectroscopy to investigate the adhesion of living adherent cells. Langmuir, 26: 8180-8186.

[7] Friedrichs, J., Helenius, J., Muller, D. J. (2010) Stimulated single-cell force spectroscopy to quantify cell adhesion receptor crosstalk. Proteomics,10: 1455-1462.

[8] Benoit, M., Gaub, H. E. (2002) Measuring cell adhesion forces with the atomic force microscope at the molecular level. Cells Tissues Organs, 172: 174-189.

[9] Hosseini, B. H., Louban, I., Djandji, D., et al. (2009) Immune synapse formation determines interaction forces between T cells and antigen-presenting cells measured by atomic force microscopy. Proc Natl Acad Sci U S A, 106: 17852-17857.

[10] Weder, G., Guillaume-Gentil, O., Matthey, N., et al. (2010) The quantification of single cell adhesion on functionalized surfaces for cell sheet engineering. Biomaterials, 31: 6436-6443. 